\title{
La dignité de l'âge
}

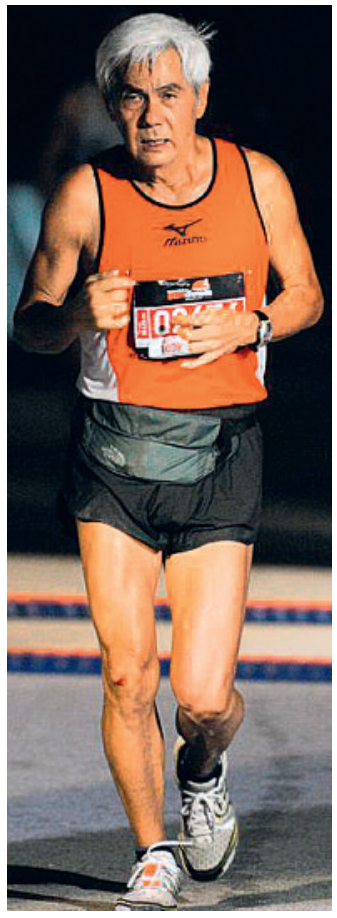

1 Balance und Newsletter 1/2009. Promotion de la santé et prévention Bâle.

2 Warum viele ältere Menschen krisenresistent sind. Entretien avec Mike Martin. Neue Zürcher Zeitung; 6/7 juin 2009.

3 Paul Parin, Tagesanzeiger. Magazin; 2009 (23).
Dans «La vieille dame indigne» Bertolt Brecht, narre les deux dernières années de vie de Mme $\mathrm{B}$. en faisant appel à deux narrateurs différents. Le titre reprend le jugement du fils qui ne peut pas comprendre que sa mère, désormais veuve, fasse passer son autonomie tardive avant les attentes de sa famille. Seul son petitfils, sans prétentions ni exigences, sera à même de comprendre pourquoi Mme B., lors des deux dernières années de sa vie - elle mourra à l'âge de 72 ans - passe son temps au cinéma, mange au restaurant plutôt que chez elle et assiste aux courses de chevaux, boit du vin et joue aux cartes. En 1965, presque 20 ans après la publication de la nouvelle, le cinéaste français René Allio en fait un film. Dans «La vieille femme indigne», Rosalie - son nom au cinéma - retombe amoureuse et s'achète une voiture au lieu de vivre une vie frugale et retirée comme il se doit pour une personne âgée qui a des héritiers.

Ce qui peut sembler une banale histoire d'émancipation n'a cependant pas pris une ride, car les attentes en matière de rôles sont toujours aussi normatives pour les personnes âgées. Aujourd'hui, la génération des seniors est au centre de la «promotion santé» ou comme le dit plus poétiquement le département de la santé du canton de Bâle-Ville, il s'agit d'explorer et de donner une forme à ce «nouveau continent de la longévité» [1]. Mme B. ou Rosalie ou quiconque, suivent à la lettre les directives et bulletins d'informations pour entraîner leur corps et leur esprit. Le vieux proverbe allemand qui dit que «comme les oiseaux chantent, les oisillons gazouillent» n'a donc plus cours. La cosmétique et la chirurgie esthétique le prônent de manière encore plus crue avec des slogans de type «jeune un jour, jeune toujours» ou «homme de fer, je le fus et je le reste». Ce dernier fait des haltères et du crawl, du jogging ou de la marche nordique, va chercher pulsomètre ou podomètre à l'office du tourisme et sait exactement quand il doit se mettre au Tai-chi et aux exercices de prévention des chutes ou à faire de l'aérobic pour le cerveau de manière à améliorer ses connexions neuronales. Beaucoup de fruits et de légumes, une planification judicieuse de l'apport en vitamines et minéraux accompagnée d'une recette de daurade aux courgettes et au safran sont à mettre dans le sac-à-dos de tout vieillard actif. Rien à redire à cela, si ce n'est que la question des coûts revient sans cesse en arrière-fond.
Etant donné que c'est surtout au cours des deux dernières années de vie que nous occasionnons le plus de frais de santé, il convient de repousser cette limite le plus loin possible. De plus, il importe de limiter la nécessité de recevoir des soins au moyen des mesures susmentionnées. La nébuleuse verbale ne permet toutefois pas de cacher la morale sous-jacente faisant poindre la mauvaise conscience. Est indigne, celui qui ne fait rien pour rester en forme et qui figure en queue de peloton dans ce marathon. La consommation obligatoire de nombreuses pommes croquantes ne donne ni accès au Paradis, ni à l'arbre de la science du bien et du mal ni droit à l'immortalité. Fitness et bien-être font certes bon ménage, mais les économies ainsi escomptées restent un vague calcul destiné aux stratèges du «carnet du lait».

Le pain et les jeux sont importants. Les autres paramètres sont analysés par le centre de gérontologie de l'Université de Zurich. Les chercheurs aimeraient connaître l'influence de la religion et de la spiritualité sur la prédisposition aux maladies. Qu'est-ce qui permet de tenir lorsque les relations rompent ou que la maladie met la vie en danger? Les questions de la qualité et du sens de la vie prendront plus d'importance à l'avenir, les rôles attribués aux personnes âgées seront remis en question et analysés de manière différenciée. Le temps où il fallait un consensus sur le meilleur rôle à un âge avancé est révolu [2]. Faire du fitness est certes une affaire sérieuse, mais elle a son prix. Le vil argent n'est jamais nommé et pourtant, il est bien clair que les mesures proposées s'adressent à une clientèle aisée, à même de s'offrir les listes d'achats établies par les experts, les recommandations de voyages, les accessoires de sport, les séminaires et les cours.

Dans l'un des derniers entretiens qu'il a donnés, on a demandé au psychanalyste Paul Parin, décédé il y a peu, comment l'on garde sa dignité avec l'âge. Parin, aveugle et très âgé, photographié assis à son bureau devant des boîtes de médicaments et un cendrier plein de mégots, répondit: «La dignité de l'âge est une notion bien abstraite. Il faut avoir des moyens matériels pour y parvenir. Il ne faut pas beaucoup d'argent, mais suffisamment, sinon s'en est fini de la dignité de l'âge» [3].

Erhard Taverna 